\title{
BMJ Open Predictors of chronic pain and level of physical function in total knee arthroplasty: a protocol for a systematic review and meta-analysis
}

\author{
Unni Olsen (D) ,,2 Maren Falch Lindberg (D) , ${ }^{1,2}$ Eva Marie-Louise Denison, ${ }^{3}$ \\ Christopher James Rose, ${ }^{3}$ Caryl Lynn Gay (1) , ${ }^{4,5}$ Arild Aamodt, ${ }^{2}$ \\ Jens Ivar Brox (1) , ${ }^{6,7}$ Øystein Skare, ${ }^{2}$ Ove Furnes, ${ }^{8,9}$ Kathryn A Lee, ${ }^{4}$ \\ Anners Lerdal (i) 5,10
}

To cite: Olsen U, Lindberg MF, Denison EM-L, et al. Predictors of chronic pain and level of physical function in total knee arthroplasty: a protocol for a systematic review and meta-analysis. BMJ Open 2020;10:e037674. doi:10.1136/ bmjopen-2020-037674

- Prepublication history and additional material for this paper are available online. To view these files, please visit the journal online (http://dx.doi. org/10.1136/bmjopen-2020037674).

Received 14 February 2020

Revised 17 June 2020

Accepted 22 July 2020
Check for updates

(c) Author(s) (or their employer(s)) 2020. Re-use permitted under CC BY-NC. No commercial re-use. See rights and permissions. Published by BMJ.

For numbered affiliations see end of article.

Correspondence to

Unni Olsen;

u.s.j.olsen@studmed.uio.no

\section{ABSTRACT}

Introduction One in five patients undergoing total knee arthroplasty (TKA) experience unchanged or worse pain and physical function 1 year after surgery. Identifying risk factors for unfavourable outcomes is necessary to develop tailored interventions to minimise risk. There is a need to review more current literature with updated methodology that addresses the limitations of earlier systematic reviews and meta-analyses. We present a Preferred Reporting Items for Systematic Review and Meta-Analysis Protocols compliant protocol for a systematic review and meta-analysis of predictors of chronic pain and impaired function after TKA.

Methods and analysis This review will include prospective longitudinal observational studies, or randomised trials (including cluster and crossover designs) that report arm-wise predictors of chronic postsurgical pain or impaired physical function at 3 months, 6 months or 12 months. A comprehensive literature search of studies published between 2000 and 2019 will be performed in Medline, Embase, CINAHL, Cochrane Library and PEDro. Blinded assessment with consensus agreement will be applied for inclusion of studies, data extraction and assessment of bias risk (Quality in Prognosis Studies tool). The co-primary outcomes, pain and impaired function, at 12 months after TKA will be analysed separately. Estimates of association between each outcome and any preoperative or intraoperative factor that may predict chronic pain or impaired physical function will be extracted from the included studies, where possible. For randomised studies, results will only be extracted from TKA arms (or the first period of crossover trials). Estimates of association from the primary evidence will be synthesised narratively, and quantitatively using multivariate meta-analysis to provide 'pooled' estimates of association. Subgroup and sensitivity analyses will be performed. Certainty of evidence for each predictor will be derived from the Grading of Recommendations Assessment, Development and Evaluation framework.

Ethics and dissemination No ethical issues are associated with this project. The results from this review will be published in peer-reviewed journals and presented at international conferences.
Strengths and limitations of this study

- The strengths of this review include a transparent protocol with rigorous and updated methodology throughout each phase of the review process, a comprehensive literature search with no limitations on predictors or language, and inclusion of only the strongest observational study designs to avoid excessive heterogeneity and a thorough description of the data analysis plan.

- Use of consistent methods for assessing the risk of bias (Quality in Prognosis Studies tool) and certainty of evidence (Grading of Recommendations Assessment, Development and Evaluation framework) is also a study strength.

- Since $95 \%$ of patients treated with total knee arthroplasty suffer from osteoarthritis, results will have high generalisability within the osteoarthritis population, but results might be less applicable to other populations, such as adults or children with rheumatoid arthritis.

- The validity of the results of this systematic review and meta-analysis will depend on the quality of the published studies included, the definitions applied for chronic pain or impaired physical function and the possible predictors included.

PROSPERO registration number CRD42018079069.

\section{INTRODUCTION}

Total knee arthroplasty (TKA) is a common surgical procedure for patients with osteoarthritis (OA) suffering from pain and impaired function. ${ }^{12}$ In the United Kingdom and the Isle of Man, nearly 100000 primary TKAs are performed annually, ${ }^{3}$ while the number for the USA is $700000 .{ }^{4}$ Despite advances in anaesthesia and the surgical field, such as implementation of fast track surgery, $20 \%$ of TKA patients experience pain and levels of physical function that remains unchanged or 
worse 1 year after surgery. ${ }^{5-7}$ Unfavourable outcomes can seriously impact patients through further deterioration in health status ${ }^{89}$ and dissatisfaction with postoperative rehabilitation and surgical outcomes. ${ }^{9-12}$ Patients who do not benefit from surgery are also more likely to undergo revision surgery, ${ }^{3} 1314$ have higher healthcare utilisation and are less likely to return to work. ${ }^{10}{ }^{15-17}$ Consequently, poor TKA outcomes represent a significant burden, on a personal level to the individual patient and family level, as well as on a socioeconomic level, with considerable healthcare resources being spent on ineffective TKA procedures. $^{18}$

One strategy to reduce the burden of poor TKA outcomes, for individual patients and society, is to gain a better understanding of the preoperative and intraoperative predictors of chronic pain and impaired function after TKA. Knowledge of preoperative and intraoperative risk factors is a fundamental first step in the development of screening tools to identify patients at high risk for chronic pain or impaired function after TKA. Identifying such patients would allow targeted and tailored interventions to be developed in order to improve patients' surgical outcomes. ${ }^{19}{ }^{20}$ Early identification of patients at high risk can also provide both patients and clinicians with more personalised information about the risks of surgery during the decision-making process when considering TKA.

During the last decade, a considerable number of hypothesised preoperative predictors of chronic pain and poor function after TKA have been investigated (figure 1). Systematic reviews and meta-analyses exist, ${ }^{21-34}$ but have yielded contradictory findings. This could be due to the use of methods that deviate from what is now understood to be a good practice, as codified by the Preferred Reporting Items for Systematic Reviews and Meta-Analysis (PRISMA) statement, ${ }^{35}$ and the recently updated Cochrane Handbook. ${ }^{36}$ The existing reviews are also more than 5 years old, ${ }^{21-27}$ and several new studies have been published in the interim.

Thus, a new synthesis of the literature that uses evidence-based methods is warranted to better inform patients, clinicians, researchers and policy-makers about risk factors for patient outcomes of chronic pain and impaired physical function after TKA. The study described in this protocol will investigate outcomes that earlier reviews did not address and use systematic review and meta-analysis tools that were not available when prior reviews were published. This systematic review and metaanalysis will be conducted according to state-of-the-art evidence-based methods (as outlined by Cochrane), and

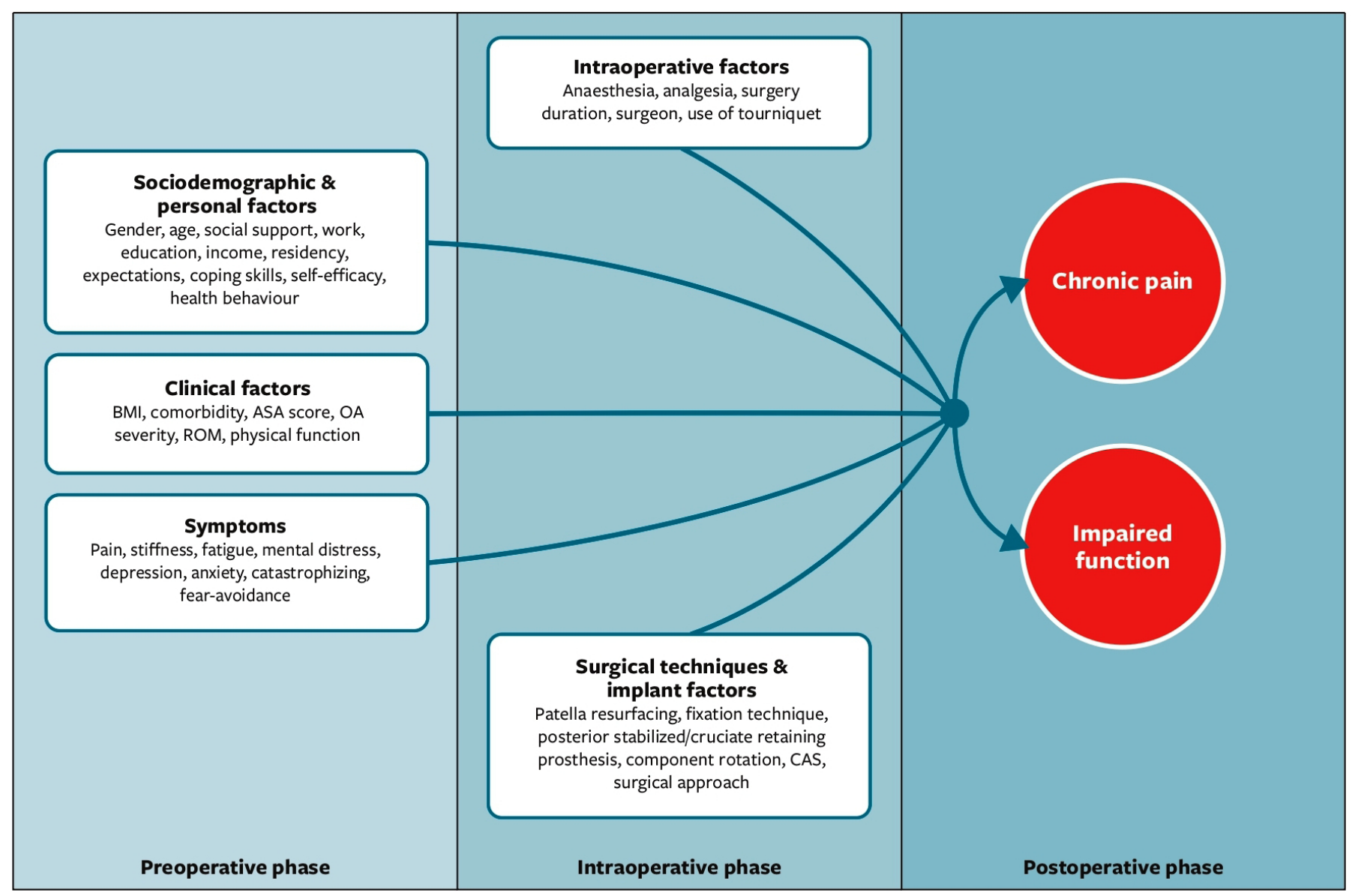

Figure 1 Hypothesised preoperative and intraoperative predictors of chronic pain and poor function after total knee arthroplasty. ASA score, The American Society of Anesthesiologists score; CAS, computer assisted surgery; OA severity, osteoarthrithis severity; ROM, range of motion. 
will cover two related, yet distinct outcomes: pain and function. Gaps in knowledge will be addressed, that will be useful for researchers in exploring areas that have previously received little research attention. Thus, the aim of this study is to conduct a systematic review and synthesis of current evidence. The result of this work will include a narrative description of the factors identified, and a statistical meta-analysis that provides point estimates and $95 \%$ CIs of the strength of association between each preoperative and intraoperative factor evaluated by the included studies, and the co-primary outcomes of chronic pain and impaired function following TKA at short-term, mediumterm and long-term follow-ups (3 months, 6 months and 12 months).

\section{METHODS AND ANALYSISS}

This systematic review and meta-analysis will include two key outcomes, chronic pain and impaired physical function, which are moderately to strongly associated, but distinct. ${ }^{37}$ Thus, chronic pain and impaired function will be assessed and reported as two separate outcomes. Our strategy is consistent with the Cochrane Handbook for Systematic Reviews of Interventions, ${ }^{36}$ which suggests that a review may start with a broad scope before being divided into more narrow reviews.

This protocol has been developed according to the Preferred Reporting Items for Systematic Review and Meta-Analysis Protocols checklist and the review will be reported according to the PRISMA guidelines. ${ }^{35}$ The described study will be guided by the biopsychosocial framework, ${ }^{38}$ found suitable for $\mathrm{OA}^{39}$ and TKA patients. ${ }^{40}$ This model takes into account the complex interplay between biological, psychological and social factors when understanding health condition and outcomes following TKA surgery for OA. The framework is operationalised in figure 1 .

\section{Eligibility criteria for considering studies in this review}

Prospective longitudinal observational studies or the TKA arm of randomised trials (including cluster and crossover designs) of OA patients undergoing primary TKA and that report at least one preoperative or intraoperative predictor of chronic postsurgical pain or impaired function (measured 3 months, 6 months or 12 months after primary TKA) will be considered for inclusion.
The data from non-TKA arms will be excluded because we are only interested in associations between predictors and outcomes in patients treated with TKA (we are not interested in predicting pain or function for other treatments, nor are we interested in the relative effects of TKA vs other interventions). For crossover trials, only the first period of the TKA arm will be included to avoid carryover effects. Studies of unicompartmental surgery, studies without separate outcome data for TKA patients, studies that lack clear pain and physical function outcome measures, retrospective studies and case-control studies will be excluded.

The eligibility criteria are pre-specified by the Population-Exposure-Outcome-Study (PEOS) design, as described below.

Population: patients 18 years or older with $\mathrm{OA}$ and scheduled for primary TKA.

Exposures: any preoperative or intraoperative factors that may predict chronic pain and impaired physical function in TKA patients.

Outcome: the two co-primary outcomes are pain and function assessed 12 months after TKA. Where possible, these outcomes will also be analysed at 3 months's and 6 month's after TKA.

Study design: a prospective longitudinal observational design, or a randomised trial design (including cluster and crossover designs).

\section{Timeline}

The timeline for the study phases is shown in table 1 . The research question has been specified, protocol details have been registered and published, the search has been performed and formal screening of the search results against eligibility criteria is in progress. Full-text inclusion is ongoing.

\section{Review question}

The review question is: 'which factors predict chronic pain and impaired physical function among patients after TKA?'.

\section{Definitions}

Chronic/persistent pain is defined as pain extending 3 months after TKA. ${ }^{41}$ Physical function refers to all body functions, activities and participation according to the

\begin{tabular}{|c|c|c|c|c|c|c|c|c|}
\hline Review question & $\begin{array}{l}\text { Register } \\
\text { review }\end{array}$ & $\begin{array}{l}\text { Search } \\
\text { strategy }\end{array}$ & $\begin{array}{l}\text { Study } \\
\text { selection }\end{array}$ & $\begin{array}{l}\text { Risk of } \\
\text { bias }\end{array}$ & $\begin{array}{l}\text { Data } \\
\text { extraction }\end{array}$ & Analysis & Certainty evidence & Publication \\
\hline $\begin{array}{l}\text { Completed } \\
30 \text { May } 2018\end{array}$ & $\begin{array}{l}\text { Completed } \\
31 \text { August } 2018\end{array}$ & $\begin{array}{l}\text { Completed } \\
1 \text { August } 2019\end{array}$ & Ongoing & Ongoing & Ongoing & Ongoing & Planned & Planned \\
\hline
\end{tabular}




\begin{tabular}{ll}
$\begin{array}{l}\text { Table } 2 \text { Outcomes and how } \\
\text { included studies }\end{array}$ \\
\hline $\begin{array}{l}\text { Measures of the chronic } \\
\text { pain outcome }\end{array}$ & $\begin{array}{l}\text { Measures of the physical } \\
\text { function outcome }\end{array}$ \\
\hline $\begin{array}{l}\text { Western Ontario and } \\
\text { McMaster Universities }\end{array}$ & $\begin{array}{l}\text { Western Ontario and } \\
\text { McMaster Universities }\end{array}$ \\
Osteoarthritis Index & Osteoarthritis Index \\
Knee Society Score & Knee Society Score \\
$\begin{array}{l}\text { Knee Injury and Osteoarthritis } \\
\text { Outcome Score }\end{array}$ & $\begin{array}{l}\text { Knee Injury and Osteoarthritis } \\
\text { Outcome Score }\end{array}$ \\
Short Form 36 & Short Form 36 \\
Oxford Knee Score & Oxford Knee Score \\
McGill Pain Questionnaire & Timed Up and Go Test \\
Brief Pain Inventory & Sit to Stand Test \\
Numerical Rating Scale & Range of Motion \\
Visual Analogue Scale & $\begin{array}{l}\text { Inertial measurement units } \\
\text { (Gait pattern) }\end{array}$ \\
\hline
\end{tabular}

International Classification of Functioning, Disability and Health framework. ${ }^{42}$

Chronic pain and impaired physical function can be measured in various ways and questionnaires (table 2), including as a continuous variable that represents a continuum of pain (eg, a score on a Visual Analogue Scale) or as a categorical variable (eg, a dichotomous variable with categorical levels of 'chronic pain' or 'no chronic pain'). Similarly, physical function can be assessed on a continuum (eg, as indicated by the Knee Injury and Osteoarthritis Outcome Score) or as different categories of function (eg, 'no problems walking', 'some problems walking' and 'confined to bed', as in EQ 5D 3L).

\section{Literature search strategy}

The search strategy was developed by two medical librarians (Gunn Kleven and Hilde Flaaten) in cooperation with the authors (UO and MFL) and with input from the experienced research team. The search strategy was designed by one research librarian (Gunn Kleven) and peer reviewed by the second research librarian (Hilde Flaaten) and first author (UO), as recommended by the Cochrane Handbook. ${ }^{36}$ A comprehensive systematic search for articles published from 1 January 2000 through 1 August 2019 was conducted (Gunn Kleven) using a combination of text words and database-specific subject headings in Medline (Ovid), Embase (Ovid), CINAHL (EBSCO), Cochrane Library and Physiotherapy Evidence Database. The search strategies were adapted to each database as presented in online supplementary material.

To capture as many relevant studies as possible, no language restrictions were applied, as recommended in Methodological Expectations of Cochrane Intervention Reviews (MECIR) standards. ${ }^{43}$ The search was limited to studies published in or after year 2000 in consideration of changes in treatment modalities since year 2000. Duplicates were removed and conference abstracts were excluded. Studies had to be available in full-text format. References were imported to EndNote V.X8 (Clarivate Analytics, Philadelphia, Pennsylvania, USA).

\section{Study selection and data extraction}

To avoid missing relevant articles, an overly inclusive approach for screening titles, abstracts and full text will be used. Publication abstracts in non-English and nonScandinavian languages will be translated and assessed for eligibility. Both screening and selecting studies for full-text review will include independent and blinded screening by two authors (UO and MFL), with consensus discussion to resolve disagreements. If consensus cannot be reached, a third reviewer will adjudicate (EM-LD). Studies that fulfil the eligibility criteria will be retained, fully translated and scrutinised for full-text assessment against eligibility criteria. A standardised data extraction form customised to the research question will be developed for extraction of data and pilot-tested on the first three included studies (table 3 ). If additional data are needed about a particular study, the corresponding and/

Table 3 Data extraction template

Data Extracted data

Publication details First author and senior author, year of publication and country of origin

Study characteristics Study design (prospective longitudinal observational design; intervention arm of a randomised trial; and intervention arm of the first period of a randomised crossover trial), source of patient recruitment, length of follow-up, sample size, statistical method and results

\section{Patients characteristics Age, sex, body mass index, ethnicity, socioeconomics and demographics}

Intervention Type of implant and anaesthetic and analgesic factors

Predictors Type of predictors and how they are measured, for example, pain by Brief Pain Inventory, depression by Hospital Anxiety Depression Scale, severity of osteoarthritis by Kellgren Lawrence Scale and direction of effect (reversed or not)

Outcome Type of pain or function outcome, how it is defined and measured (table 2); the unit of analysis used (patient or cluster)

Measure of association Analysis type (eg, linear regression or correlation coefficient), estimate (ie, numerical result) and (one per predictor) precision (eg, $\mathrm{Cl}, \mathrm{SE}, \mathrm{p}$ value) 
or senior authors of the publication will be contacted to obtain more detail.

It is anticipated that included studies may present multiple results for each predictor (eg, several regression models resulting from stepwise model-building procedures). The data will be extracted for the model or analysis specified as the primary analysis in the study protocol; if no suitable model or analysis is specified, we will extract data for the model or analysis favoured by the study's authors and presented as the 'main result' or 'full model' (eg, the model with the best goodness of fit criteria, such as Akaike's information criterion). ${ }^{44}$ A consensus-based approach will be used to determine which result is favoured by a study (ie, two authors performing data extraction must agree; in the event of disagreement, a third author will adjudicate). Acquisition and analysis of individual patient-level data (IPD) is not planned. For example, we will not re-analyse IPD with respect to our own definitions of the co-primary outcomes.

Studies may use different names for the same predictor. Predictors will be considered to be the same, if they are measured using the same method (eg, instrument), or if the methods of measurement are judged to assess the same construct (eg, anxiety) by two authors (in the event of disagreement, a third author will adjudicate).

Variables of interest measured before TKA and included in pre-specified or 'main' analyses will be extracted and included in meta-analyses. Studies may report estimates adjusted for variables such as age, sex and presurgical pain, as well as unadjusted estimates. Because we are interested in predictors' independent value over and above other predictors, we will extract estimates from adjusted models (even if some available predictors were excluded from the 'final' model, eg, as a result of a model-building approach; estimates for omitted predictors will be treated as missing as described below).

For randomised trials, data will be extracted for the TKA arm and treated in the same way as longitudinal observational data. For crossover trials, data will be extracted from the first period of the TKA arm and will be treated similarly. Because cluster designs will be included, we will extract the unit of analysis used for all studies (patient vs cluster). If studies that use a cluster design report results that do not account for possible cluster effects, we will impute results that adjust for clustering where feasible; if it is not feasible to adjust for clustering, we will judge the study to have a high risk of bias (see below). It is anticipated that publications that report randomised designs may not provide arm-wise results. We will contact authors of such studies and request the required data; studies will be excluded from synthesis if data are not received within 4 weeks of request.

If associations are reported for multiple levels of an ordinal predictor (eg, associations between pain and overweight vs normal weight, and obese vs normal weight), we will extract data for the most extreme comparison (obese vs normal weight, in this example).

\section{Measures of association}

Based on a scoping exercise, studies may report estimates of association between categorical or continuous predictors and outcomes. Association may be quantified using odds or risk ratios, linear model coefficients (including differences) and correlation coefficients. Furthermore, base cases of categorical variables may vary, as may directions of association. We will define canonical directions of association and measure association using the correlation coefficient, which is defined to be invariant under linear transformations of predictor and outcome variables and, under reasonable assumptions, can be imputed for all combinations of dichotomous and continuous predictors and outcomes (see online supplementary methods). If it is necessary to impute ORs from risk ratios, we will assume a baseline probability of postsurgical pain and impaired function of 20\%. Meta-analysis will be performed by transforming correlation coefficients using Fisher's z transformation. ${ }^{45}$ This approach is similar to that used in a previous review on this topic by Lewis et al. ${ }^{27}$

\section{Methodological quality}

To systematically evaluate study quality and to reach consensus in a transparent manner, the Quality in Prognosis Studies (QUIPS) tool will be used to assess risk of bias in the individual studies. ${ }^{46}$ QUIPS tool addresses six domains where bias may occur in prognostic studies: study participation, attrition, prognostic factor measurement, outcome measurement, confounding and statistical analysis and reporting. ${ }^{36}$ Two authors (UO and MFL) must agree, otherwise a third reviewer (EM-LD) will adjudicate.

\section{Dealing with missing data}

Study authors will be contacted if there is a need for additional details about unpublished data, as recommended by the Cochrane Handbook. ${ }^{36}$ Requests will be sent to a study's corresponding author and the first or senior author if the corresponding author cannot be contacted. In addition, critical appraisal will be carried out and reported regarding study participant attrition, losses to follow-up or withdrawal and any issues regarding missing data or imputation methods (eg, last observation carried forward).

It is anticipated that not all included studies will report associations between outcomes and all predictors that have been studied in the literature as a whole (ie, the nature of the research question suggests that estimates for some predictors may be missing for most studies). This form of missing data will be addressed in a metaanalysis, as described in the data synthesis section.

It is assumed that some included studies will report point estimates but not exact statements of uncertainty on those estimates (eg, some studies may report results as 'statistically significant' rather than providing an exact $\mathrm{p}$ value or $\mathrm{CI}$ ). A conservative approach will be used in which 'worst case' standard errors will be imputed: results reported as 'statistically significant' $(\mathrm{eg}, \mathrm{p} \leq 0.05)$ will be imputed to have standard errors consistent with $\mathrm{p}=0.05$; 
results reported as 'not statistically significant' (eg, $\mathrm{p}>0.05$ ) will be imputed to have standard errors consistent with $\mathrm{p}=0.99$.

\section{Data synthesis}

A narrative analysis of the results will be conducted for all included studies for the two co-primary outcomes at 12 months. Meta-analyses will be performed for the two co-primary outcomes of chronic pain and impaired physical function assessed 3 months and 6 months after the surgery, if possible.

Where possible, we will perform quantitative data synthesis following the guidance of the most recent version of the Cochrane Handbook available at the time of the analysis. ${ }^{36}$ If meta-analysis cannot be performed, we will conduct a narrative analysis.

It is anticipated that predictors may be correlated and that there may be important differences in the methods used to quantify associations between outcomes and predictors (ie, while the methods used in the included studies will attempt to measure compatible constructs, they are likely to be sufficiently different that we expect heterogeneity). Multivariate meta-analysis will, therefore, be performed for each outcome using a randomeffects model. Analyses will be performed using Stata V.16 (StataCorp LLC, College Station, Texas, USA) and the MVMETA command or $\mathrm{R}$ and the metafor package. ${ }^{47-49}$ Missing point estimates for predictors not included in individual studies will be handled using the standard procedures defined by the software used. For example, the MVMETA command models missing point estimates as zeros and accounts for uncertainty using very large variances (ie, missing point estimates could plausibly take any value). Within-study correlations will be assumed to be unknown and the 'overall correlation model' of Riley et $a \tilde{l}^{\tilde{0}}$ will be used. An unstructured between-study covariance matrix will be assumed. If negligible correlations are inferred, we may report univariate meta-analyses by predictor.

\section{Assessment of non-reporting bias and small-study effects}

Non-reporting bias and small study effects will be assessed following the approach outlined by Sterne et $a l^{51}$ For each predictor supported by at least 10 results, contourenhanced funnel plots will be constructed by plotting Fisher's Z against its SE. Funnel plot asymmetry will be judged visually and tested using Egger's regression-based test (at the $\alpha=0.05$ level) assuming random effects. Predictors for which asymmetry is suspected will be reported with consideration for the possible causes of asymmetry. In particular, asymmetry will not be definitively attributed to non-reporting bias because it may have other explanations. Fixed-effects and random-effects meta-analysis estimates will not be compared because we anticipate heterogeneity and, therefore, judge that the fixed-effects model is inappropriate. Predictors will not be excluded from meta-analysis on the basis of suspected asymmetry but will be downgraded for certainty of evidence (see below).

\section{Assessment of heterogeneity}

Clinical and methodological heterogeneity will be evaluated subjectively. We will interpret $\mathrm{I}^{2}$ values following guidelines in the Cochrane Handbook ${ }^{36}$ and Grading of Recommendations Assessment, Development and Evaluation (GRADE) framework. ${ }^{52}$ Exploratory analyses may be performed to attempt to explain any substantial heterogeneity.

\section{Subgroup analysis}

Exploratory subgroup meta-analyses will be performed for the two co-primary outcomes with respect to study design, type of outcome measurement and intervention (eg, type of implant). Subgroup analyses will only be performed if at least 10 studies can be included in each subgroup.

\section{Sensitivity analysis}

We will perform exploratory sensitivity analyses for the two co-primary outcomes. For each of the six QUIPS tool's bias domains, we will perform meta-analyses by excluding studies judged to be at high risk of bias and compare meta-analysis results to those obtained when all studies are included. We will compare meta-analysis results when pain and impaired physical function are modelled as two separate outcomes versus a single multivariate outcome (ie, a single multivariate model of the two co-primary outcomes will also be fitted). We will assess the influence of individual studies on meta-analytical results via leaveone-out analysis.

\section{Certainty of evidence using the GRADE framework}

The certainty of evidence for each prognostic factor will be derived using the GRADE framework for prognostic studies. ${ }^{46} 5354$ Study limitations, indirectness, inconsistency, imprecision, publication bias, magnitude of association, dose-response gradient and plausible confounding affecting confidence will be evaluated. ${ }^{46}$ The overall certainty of evidence will be rated as high, moderate, low or very low. GRADE ratings will be assigned by two of the authors (UO and MFL), issues will be discussed to arrive at consensus and if not, a third reviewer (EM-LD) will adjudicate.

\section{Presentation and interpretation of results}

Search strategy results will be presented, as well as characteristics of included studies, descriptive data for the eligibility criteria according to the PEOS design template data extraction form, data dictionary document, judgement of risk of bias by QUIPS and the GRADE evidence profile.

Funnel plots will be used for assessing presence of publication bias for the two separate outcomes. The meta-analytical result for each outcome will be presented as forest plots. For example, one forest plot will present meta-analytical point estimates and $95 \%$ CI and prediction intervals for the predictors, ${ }^{55}$ with predictors ordered by 
probability of being the best predictor (eg, via the 'pbest' option of MVMETA); additional forest plots may show results for each predictor, showing the results extracted from the included studies and the meta-analytical point estimates and $95 \% \mathrm{CI}$ and prediction intervals.

Following MECIR standards, ${ }^{43}$ meta-analytical results will be re-expressed as correlation coefficients to facilitate interpretation. We will provide layperson interpretations of these in summary of findings' tables using Cohen's labels 'small' (correlation=0.1), 'weak' (0.3) and 'large' (0.5).

Meta-analytical results will be presented in a summary of findings table for each outcome ${ }^{56}$ with columns for: predictor; meta-analytical result (Fisher's $\mathrm{Z}$ and its $95 \%$ CI); re-expressed Fisher's Z and its 95\% CI; a layperson interpretation of the magnitude of association; number of participants (and clusters, where appropriate) and number of studies; certainty of evidence (GRADE) and comments.

Subgroup and sensitivity analyses, and meta-analyses of predictors of chronic pain and impaired physical function measured at 3 months and 6 months after TKA, will be narratively summarised from any available reported data. Tables and figures for these analyses will be presented in an appendix.

Factors identified as being associated with the co-primary outcomes will not be interpreted to cause those outcomes because the included study designs and planned analyses do not allow causal inferences to be made.

\section{Deviations from protocol}

Deviations from this protocol will be reported and justified.

\section{Patient and public involvement}

No patients will be involved in this review.

\section{Strengths and limitations}

By following the Cochrane Handbook's method recommendations ${ }^{36}$ throughout each phase, we aim to achieve a high-quality review that will be of importance to patients, clinicians, researchers and policy-makers. To provide reliable data to address the review's aim and to avoid excessive heterogeneity caused by different study designs that might affect robustness of the review's results or introduce high risk of bias, only prospective longitudinal studies or the intervention arm of randomised trials will be included. No language limitations will be applied so that relevant studies are not excluded, thereby increasing precision of the findings and maximising generalisability. As a result, widespread application of the study results is expected. Although postoperative predictors may be important for non-improvement after TKA surgery, this will not be covered by this study's aim. QUIPS and GRADE will be used to assess risk of bias for individual studies and assess certainty of evidence of included studies. Since 95\% of patients treated with TKA suffer from OA, results will have high generalisability within the OA population, but might be less applicable to other populations, such as patients with rheumatoid arthritis. The strict eligibility criteria exclude studies that only report outcomes after 1 year; most registered studies will be rejected as they are less likely to be prospective. Results must be interpreted based on this study context. We might find that predictors and outcomes are measured quite differently across studies. However, an effect size for a meta-analysis creates a standardised measure so the actual measure scaling is not relevant and thus a meta-analysis is perfect for reviewing studies that use different measures for the same conceptual outcome.

\section{Ethics and dissemination}

Primary data will not be collected, thus ethical approval is not required. Results will be presented at international conferences, and findings will be published in peerreviewed high-impact journals and a doctoral thesis.

\section{User involvement}

Members from the user board at Lovisenberg Diaconal Hospital, Jan Otto Veiseth and Richard Madsen, have been contributing to the relevance and significance of the protocol's content.

\section{Author affiliations}

${ }^{1}$ Department of Nursing Science, Institute of Health and Society, Faculty of Medicine, University of Oslo, Oslo, Norway

${ }^{2}$ Department of Orthopaedic Surgery, Lovisenberg Diaconal Hospital, Oslo, Norway ${ }^{3}$ Division for Health Services, Norwegian Institute of Public Health, Oslo, Norway ${ }^{4}$ Department of Family and Health Care Nursing, University of California, San Francisco, California, USA

${ }^{5}$ Department of Patient Safety and Research, Lovisenberg Diaconal Hospital, Oslo, Norway

${ }^{6}$ Department of Physical Medicine and Rehabilitation, Oslo University Hospital, Oslo, Norway

${ }^{7}$ Institute of Clinical Medicine, Faculty of Medicine, University of Oslo, Oslo, Norway ${ }^{8}$ Norwegian Arthroplasty Register, Haukeland University Hospital, Bergen, Norway ${ }^{9}$ Department of Orthopaedic Surgery, University of Bergen, Bergen, Hordaland, Norway

${ }^{10}$ Department of Interdisciplinary Health Sciences, Institute of Health and Society, Faculty of Medicine, University of Oslo, Oslo, Norway

\section{Twitter Anners Lerdal @AnnersLerdal}

Acknowledgements The authors thank the members from the user board, Richard Madsen and Jan Otto Veiseth, for their contributions. We are thankful for the work of the medical librarians, Gunn Kleven and Hilde Flaaten, and Eira Taksdal for her assistance with the layout of the figure.

Contributors UO, MFL, EM-LD, CJR, CLG and AL contributed to the development of the protocol. UO and EM-LD were responsible for the methods section, and CJR for the statistical descriptions and the document on supplementary methods. UO wrote the manuscript. All authors, MFL, EM-LD, CJR, CLG, AA, JIB, ØS, UO, OF, KA and $A L$, provided feedback on the study design and have read and approved the final manuscript. AL is guarantor of the study.

Funding This work is supported by funding from the Norwegian Research Council of Norway (grant \#287816) and the South-Eastern Regional Health Authority (grant \#2018060).

Competing interests CJR reports personal fees from Oncolmmunity AS. He has a patent and patent application with no relevance to this study.

Patient and public involvement Patients and/or the public were involved in the design, or conduct, or reporting, or dissemination plans of this research. Refer to the Methods section for further details.

Patient consent for publication Not required. 
Provenance and peer review Not commissioned; externally peer reviewed.

Open access This is an open access article distributed in accordance with the Creative Commons Attribution Non Commercial (CC BY-NC 4.0) license, which permits others to distribute, remix, adapt, build upon this work non-commercially, and license their derivative works on different terms, provided the original work is properly cited, appropriate credit is given, any changes made indicated, and the use is non-commercial. See: http://creativecommons.org/licenses/by-nc/4.0/.

\section{ORCID iDs}

Unni Olsen http://orcid.org/0000-0003-0474-5985

Maren Falch Lindberg http://orcid.org/0000-0003-2074-2071

Caryl Lynn Gay http://orcid.org/0000-0002-6865-6335

Jens Ivar Brox http://orcid.org/0000-0002-2507-1812

Anners Lerdal http://orcid.org/0000-0002-7144-5096

\section{REFERENCES}

1 Fingar K, Stocks C, Weiss A. Most Frequent Operating Room Procedures Performed in U.S. Hospitals, 2003-2012: Statistical Brief \#186. In: Healthcare cost and utilization project (HCUP) statistical Briefs. Agency for Health Care Policy and Research Healthcare, 2014. https://www.ncbi.nlm.nih.gov/books/NBK274246/?otool= bibsys

2 Price AJ, Alvand A, Troelsen A, et al. Knee replacement. Lancet 2018;392:1672-82

3 National joint registry of England W, Northern Ireland and the Isle of man. National joint registry 16th annual report 2019, 2019. Available: https://reports.njrcentre.org.uk/Portals/0/PDFdownloads/NJR\% 2016th\%20Annual\%20Report\%202019.pdf [Accessed 10 Jun 2019]

4 Inacio MCS, Paxton EW, Graves SE, et al. Projected increase in total knee arthroplasty in the United States - an alternative projection model. Osteoarthritis Cartilage 2017;25:1797-803.

5 Beswick AD, Wylde V, Gooberman-Hill R, et al. What proportion of patients report long-term pain after total hip or knee replacement for osteoarthritis? A systematic review of prospective studies in unselected patients. BMJ Open 2012;2:e000435.

6 Lindberg MF, Miaskowski C, RustøEn T, et al. Factors that can predict pain with walking, 12 months after total knee arthroplasty. Acta Orthop 2016;87:600-6.

7 Petersen KK, Simonsen O, Laursen MB, et al. Chronic postoperative pain after primary and revision total knee arthroplasty. Clin J Pain 2015;31:1-6.

8 Lindberg MF, Rustøen T, Miaskowski C, et al. The relationship between pain with walking and self-rated health 12 months following total knee arthroplasty: a longitudinal study. BMC Musculoskelet Disord 2017;18:1-10.

9 Clement ND, Bardgett M, Weir D, et al. Increased symptoms of stiffness 1 year after total knee arthroplasty are associated with a worse functional outcome and lower rate of patient satisfaction. Knee Surg Sports Traumatol Arthrosc 2019;27:1196-203.

10 Kuijer PPFM, Kievit AJ, Pahlplatz TMJ, et al. Which patients do not return to work after total knee arthroplasty? Rheumatol Int 2016;36:1249-54.

11 Kahlenberg CA, Nwachukwu BU, McLawhorn AS, et al. Patient satisfaction after total knee replacement: a systematic review. Hss J 2018;14:192-201.

12 Gunaratne R, Pratt DN, Banda J, et al. Patient Dissatisfaction following total knee arthroplasty: a systematic review of the literature. $J$ Arthroplasty 2017;32:3854-60.

13 Dalury DF, Pomeroy DL, Gorab RS, et al. Why are total knee arthroplasties being revised? J Arthroplasty 2013;28:120-1.

14 Maradit Kremers H, Kremers WK, Berry DJ, et al. Patient-Reported outcomes can be used to identify patients at risk for total knee arthroplasty revision and potentially Individualize postsurgery followup. J Arthroplasty 2017;32:3304-7.

15 Tilbury C, Schaasberg W, Plevier JWM, et al. Return to work after total hip and knee arthroplasty: a systematic review. Rheumatology 2014;53:512-25.

16 Kallala RF, Vanhegan IS, Ibrahim MS, et al. Financial analysis of revision knee surgery based on NHS tariffs and hospital costs: does it pay to provide a revision service? Bone Joint J 2015;97B:197-201.

17 Maradit Kremers H, Visscher SL, Moriarty JP, et al. Determinants of direct medical costs in primary and revision total knee arthroplasty. Clin Orthop Relat Res 2013;471:206-14.

18 Breivik H, Eisenberg E, O'Brien T, et al. The individual and societal burden of chronic pain in Europe: the case for strategic prioritisation and action to improve knowledge and availability of appropriate care. BMC Public Health 2013;13:1229.

19 Wylde V, Beswick AD, Dennis J, et al. Post-Operative patientrelated risk factors for chronic pain after total knee replacement: a systematic review. BMJ Open 2017;7:e018105.

20 Beswick AD, Wylde V, Gooberman-Hill R. Interventions for the prediction and management of chronic postsurgical pain after total knee replacement: systematic review of randomised controlled trials. BMJ Open 2015;5:e007387.

21 Santaguida PL, Hawker GA, Hudak PL, et al. Patient characteristics affecting the prognosis of total hip and knee joint arthroplasty: a systematic review. Can J Surg 2008;51:428-36.

$22 \mathrm{He} \mathrm{J}-\mathrm{Y}$, Jiang L-S, Dai L-Y. Is patellar resurfacing superior than nonresurfacing in total knee arthroplasty? A meta-analysis of randomized trials. Knee 2011;18:137-44.

23 Li S, Chen Y, Su W, et al. Systematic review of Patellar resurfacing in total knee arthroplasty. Int Orthop 2011;35:305-16.

24 Vissers MM, Bussmann JB, Verhaar JAN, et al. Psychological factors affecting the outcome of total hip and knee arthroplasty: a systematic review. Semin Arthritis Rheum 2012;41:576-88.

25 van Jonbergen H-PW, Reuver JM, Mutsaerts EL, et al. Determinants of anterior knee pain following total knee replacement: a systematic review. Knee Surg Sports Traumatol Arthrosc 2014;22:478-99.

26 Burns LC, Ritvo SE, Ferguson MK, et al. Pain catastrophizing as a risk factor for chronic pain after total knee arthroplasty: a systematic review. J Pain Res 2015;8:21-32.

27 Lewis GN, Rice DA, McNair PJ, et al. Predictors of persistent pain after total knee arthroplasty: a systematic review and meta-analysis. Br J Anaesth 2015;114:551-61.

28 Lungu E, Vendittoli P-A, Desmeules F. Preoperative determinants of patient-reported pain and physical function levels following total knee arthroplasty: a systematic review. Open Orthop J 2016;10:213-31.

29 Alattas SA, Smith T, Bhatti M, et al. Greater pre-operative anxiety, pain and poorer function predict a worse outcome of a total knee arthroplasty. Knee Surg Sports Traumatol Arthrosc 2017;25:3403-10.

30 Harmelink KEM, Zeegers AVCM, Hullegie W, et al. Are there prognostic factors for one-year outcome after total knee arthroplasty? A systematic review. J Arthroplasty 2017;32:3840-53.

31 Duan G, Liu C, Lin W, et al. Different factors conduct anterior knee pain following primary total knee arthroplasty: a systematic review and meta-analysis. J Arthroplasty 2018;33:1962-71.

32 Migliorini F, Eschweiler J, Niewiera M, et al. Better outcomes with patellar resurfacing during primary total knee arthroplasty: a metaanalysis study. Arch Orthop Trauma Surg 2019;139:1445-54.

33 Sorel JC, Veltman ES, Honig A, et al. The influence of preoperative psychological distress on pain and function after total knee arthroplasty: a systematic review and meta-analysis. Bone Joint J 2019;101-B:7-14.

34 Youlden DJ, Dannaway J, Enke O. Radiographic severity of knee osteoarthritis and its relationship to outcome post total knee arthroplasty: a systematic review. ANZ J Surg 2020;90:237-42.

35 Moher D, Shamseer L, Clarke M, et al. Preferred reporting items for systematic review and meta-analysis protocols (PRISMA-P) 2015 statement. Syst Rev 2015;4:1.

36 Higgins JPT, Thomas J, Chandler J. Cochrane Handbook for systematic reviews of interventions. version 6.0. Cochrane, 2019. www.training.cochrane.org/handbook

37 Dowsey MM, Nikpour M, Dieppe P, et al. Associations between pre-operative radiographic changes and outcomes after total knee joint replacement for osteoarthritis. Osteoarthritis Cartilage 2012;20:1095-102

38 Engel GL. The need for a new medical model: a challenge for biomedicine. Science 1977;196:129-36.

39 Hunt MA, Birmingham TB, Skarakis-Doyle E, et al. Towards a biopsychosocial framework of osteoarthritis of the knee. Disabil Rehabil 2008;30:54-61.

40 Lindberg MF. Factors that predict the trajectories of acute pain, pain with walking, and self-rated health after total knee arthroplasty, 2017.

41 Werner MU, Kongsgaard UE. I. defining persistent post-surgical pain: is an update required? $\mathrm{Br} J$ Anaesth 2014;113:1-4.

42 World Health Organization. Towards a common language for functioning, disability, and health (ICF). The International classification of functioning, disability and health, 2002.

43 Higgins JPT, Lasserson T, Chandler J, et al. Methodological expectations of Cochrane intervention reviews (MECIR. London: Cochrane Library, 2019. http://methods.cochrane.org/ methodological-expectations-cochrane-intervention-reviews

44 Akaike $\mathrm{H}$. A new look at the statistical model identification. IEEE Trans Automat Contr 1974;19:716-23. 
45 Borenstein M, Hedges LV, Higgins JP, et al. Introduction to metaanalysis. John Wiley \& Sons, 2009.

46 Hayden JA, van der Windt DA, Cartwright JL, et al. Assessing bias in studies of prognostic factors. Ann Intern Med 2013;158:280-6.

47 White IR. Multivariate Random-effects meta-analysis. Stata J 2009;9:40-56.

48 Viechtbauer W. Conducting Meta-Analyses in R with the meta for Package. J Stat Softw 2010;36.

49 R Core Team. R: a language and environment for statistical computing. $R$ foundation for statistical computing. Vienna: R Foundation for Statistical Computing, 2018. www.R-project.org/

50 Riley RD, Thompson JR, Abrams KR. An alternative model for bivariate random-effects meta-analysis when the within-study correlations are unknown. Biostatistics 2008;9:172-86.

51 Sterne JAC, Sutton AJ, loannidis JPA, et al. Recommendations for examining and interpreting funnel plot asymmetry in meta-analyses of randomised controlled trials. BMJ 2011;343:d4002.
52 Schünemann HBJ, Guyatt G, Oxman A. Grade Handbook for grading quality of evidence and strength of recommendations: the grade Working group, 2013. Available: https://www.guidelinedevelopment. org/handbook [Accessed 3 Jun 2020].

53 Huguet A, Hayden JA, Stinson J, et al. Judging the quality of evidence in reviews of prognostic factor research: adapting the grade framework. Syst Rev 2013;2:71

54 lorio A, Spencer FA, Falavigna M, et al. Use of grade for assessment of evidence about prognosis: rating confidence in estimates of event rates in broad categories of patients. BMJ 2015;350:h870:1-8.

55 Higgins JPT. Prediction intervals. In: Borenstein M, Hedges LH Rothstein HR, eds. Introduction to meta-analysis. 1 ed. John Wiley \& Sons, 2009: 127-33.

56 Guyatt G, Oxman AD, Akl EA, et al. Grade guidelines: 1. IntroductionGRADE evidence profiles and summary of findings tables. J Clin Epidemiol 2011;64:383-94. 